\title{
Le don de sperme
}

\author{
Jean-Loup CLEMENT
}

CECOS, Faculté de Médecine, Lyon

\section{RESUME}

Le recrutement des donneurs de sperme constitue une difficulté permanente depuis la création des CECOS. Les donneurs doivent toujours être sollicités soit directement, soit indirectement. Le nombre limité de grossesses pour un même sperme oblige les CECOS à recruter toujours de nouveaux donneurs ; ils ne peuvent donc pas disposer d'une population à solliciter périodiquement.

Le don de sperme, comme ceux d'autres produits humains, doit être librement consenti. Sa finalité est de permettre la conception d'enfants en dehors de l'organisation familiale du donneur. Cette donnée motive les hommes qui acceptent d'être donneurs mais en démobilise le plus grand nombre. C'est pourquoi la publicité pour le don de sperme s'impose pour favoriser un large recrutement auprès d'une population tout-venant.

Mot-clefs : don de sperme, recrutement des donneurs, publicité.

\section{INTRODUCTION}

Depuis la création des Centres d'Etude et de Conservation des Eufs et du Sperme humains (CECOS) en 1973, le recrutement des donneurs constitue une difficulté permanente à laquelle se heurte chaque centre qui pratique des Inséminations Artificielles avec Donneur (IAD). Les donneurs doivent toujours être sollicités soit directement, soit indirectement. La sollicitation directe est réalisée par les médecins, les couples en attente d'IAD. La sollicitation indirecte s'effectue par voie d'articles rédactionnels et de publicité dans la presse écrite, des reportages et émissions dans la presse audiovisuelle ainsi que l'envoi de messages à des adhérents d'organismes, par exemple les mutuelles complémentaires de santé.

La cause principale du manque de donneurs de sperme réside dans le fait que la finalité du don est la conception d'enfants. Le don de sperme ne peut être assimilé à celui d'autres produits humains (sang, moelle osseuse) qui eux contribuent seulement à la guérison d'une personne malade. L'accord passé entre le CECOS et le donneur est l'utilisation de son sperme à des fins d'insémination pour les femmes qui le demandent. Même si ce dernier n'a pas connaissance de la destination de son sperme ni des résultats obtenus, il peut imaginer que son sperme a servi à la conception de plusieurs enfants. C'est à partir de cette spécificité que s'organise le don de sperme. Le bénéfice qu'en tirent les uns (les donneurs) se renverse pour les autres, c'est-à-dire pour ceux qui refusent que des enfants naissent, de leur fait, ailleurs que dans leur propre famille.

La sollicitation des hommes en vue du don de sperme est impérative. Nous proposons ici d'examiner la population des donneurs, de réfléchir sur les résistances au don et d'étudier quels axes publicitaires sont à privilégier pour permettre un meilleur recrutement. 


\section{LES CONDITIONS DU DON}

Les CECOS ont établi des conditions de sélection des donneurs qui sont au nombre de trois : les hommes doivent être pères, en bonne santé et âgés de quarante-cinq ans au plus. La paternité préalable du donneur présume de sa fertilité et du pouvoir fécondant de son sperme, ce qui "répond tout d'abord à un souci d'efficacité et de sécurite", [4] selon G. David, biologiste, fondateur des CECOS. Les conditions pour être donneur sont strictes au regard de ce qu'elles étaient avant la création des CECOS, c'est-à-dire lorsque l'insémination artificielle était pratiquée de façon plus ou moins officielle dans des cabinets médicaux. G. David rappelle que le donneur était "un jeune célibataire que l'on motivait uniquement par une rétribution" et qui "était jusqu'alors ignoré et même méprisé du fait de la vénalité de sa démarche" [4]. G. David poursuit : "Nous avons tout d'abord recherché une réhabilitation du donneur". La paternité préalable, la gratuité et l'anonymat du don apparaissent comme des valeurs qui annulent la vénalité de la démarche. Cependant elles occultent les bénéfices secondaires que le donneur tire de son geste car l'accent est d'abord mis sur sa situation matrimoniale : il est avant tout un père de famille.

Dans cette entreprise de "réhabilitation", la notion de "don de couple à couple" s'est imposée pour devenir un véritable slogan repris par les médecins des CECOS et largement entretenu dans le discours médiatique. La notion de don de "couple à couple" présente une ambiguiité difficile à dissiper. Elle tente de faire croire que le don de sperme provient d'un couple alors qu'il est avant tout celui d'un homme. Ce dernier donne réellement et symboliquement un produit qui ne peut faire d'enfant que s'il est inséminé et provoque une grossesse. La partenaire est présente dans la mesure où elle est la mère des enfants du donneur et que ces derniers risquent d'avoir des demifrères ou søurs biologiques en dehors de la cellule familiale. Réellement, la femme ne donne rien, symboliquement, elle autorise le geste de son mari. Le slogan "don de couple à couple" accentue par ailleurs le fantasme du "don de sperme vécu comme un don d'enfant" [6] tel que le révèle une étude auprès de donneurs potentiels.

La notion de "don de couple à couple" sert à atténuer le fantasme d'adultère présent dans la pratique de l'IAD. Les demandeurs d'IAD comme les donneurs transgressent l'ordre de l'alliance en vigueur dans notre système social. L'enfant est conçu dans un couple et si l'un des deux partenaires procrée hors de ce couple établi, l'enfant est réputé adultérin. Le donneur ne se trouve pas dans la situation réelle de l'adultère, mais son acte, que l'institution médicale favorise, présente des similitudes. En souhaitant associer la femme et l'homme dans le don, les investigateurs de l'IAD ont tenté d'atténuer l'image de l'adultère.

L'âge des donneurs a été ramené récemment (juin 1993) de cinquante-cinq ans à quarante-cinq ans. Cette décision de la fédération des CECOS est intervenue suite aux conclusions des différents travaux de biologistes et de généticiens dans lesquels les auteurs démontrent que "le vieillissement de l'âge paternel est responsable de mutations autosomiques dominantes à l'origine de différentes malformations" [1] Cette décision a pour effet immédiat d'écarter environ 5\% de donneurs. Baisser l'âge maximum des donneurs a aussi un effet sur l'image de marque des CECOS. Les critiques du public (journalistes, par exemple) ne manquent pas sur l'acceptation de donneurs en âge d'être grand-pères, ce qui accentue l'inégalité des possibilités de procréation entre les hommes et les femmes.

L'interrogatoire médical du donneur permet d'évaluer s'il n'est pas porteur de malformations génétiques suceptibles d'entraîner une maladie grave ou incurable chez un enfant. Les examens biologiques permettent d'éliminer des donneurs porteurs d'infections (syphilis, chlamydiæ) ou de virus (VIH, hépatite). 


\section{LES MOTIVATIONS AU DON DE SPERME}

Une étude [2] rétrospective sur les donneurs du CECOS-Lyon nous a permis de mieux cerner les caractéristiques sociologiques de cette population. L'âge moyen des donneurs est de trente-quatre ans ; ils exercent une profession libérale, sont cadres supérieurs ou techniciens pour 58\% d'entre eux. Ils ont en moyenne 2,16 enfants (contre 1,8 dans la population générale). Ils sont issus d'une famille de 4,2 enfants en moyenne, alors que les recensements de la population française depuis quarante ans font apparaître que les familles de quatre enfants constituent seulement $5 \%$ de la population globale des familles. L'âge moyen du dernier enfant au moment du don est de 5,2 ans. Ce dernier chiffre confirme que la décision du don se situe au moment où les propres enfants du donneur sont sortis de la période de la petite enfance. Il tend aussi à confirmer l'hypothèse selon laquelle le don de sperme est la mise en acte du désir de procréer sans que cet enfant s'inscrive dans la génération directe du donneur. Le don de sperme exprime la séparation entre engendrement et reproduction : faire un enfant à soi et donner son sperme à d'autres.

Notre étude confirme également que les donneurs sont particulièrement sensibilisés par la question de l'enfance et de la reproduction. Ils sont issus de familles nombreuses, ont plus d'enfants que les hommes de leur génération mais moins que leurs parents. Le don apparaît alors comme une possibilité d'égaler, voire de dépasser, le nombre d'enfants réalisé par les parents.

Une autre étude [3] effectuée à partir d'interviews de donneurs confirme que le fantasme d'inceste entre enfants consanguins (ceux du donneur et ceux conçus à partir du don) est nié par les donneurs. Par opposition, ceux qui redoutent cette rencontre hypothétique refusent de donner leur sperme. Lorsque nous demandons aux futurs vasectomisés s'ils souhaitent être donneurs, leur refus est expliqué par ce fantasme d'inceste qu'ils ne peuvent dépasser.

Le don de sperme est unique dans la vie du donneur. La règle est qu'il ne se répétera pas. Les bénéfices sont de l'ordre de l'intime dans le sens où le don s'inscrit dans la dynamique interne du sujet. Deux explications pour illustrer à la fois les difficultés et le "plaisir" provoqués par le don de sperme.

Le prélèvement du sperme par masturbation est prescrit par l'institution médicale. C'est l'acte qui rappelle l'interdit parental sur la sexualité auquel est rattaché un sentiment de culpabilité pour l'enfant. C'est aussi celui qui ne sert pas à la procréation. Pour les uns, la prescription médicale permet de lever la culpabilité. Pour les autres, même si l'acte est conflictuel, ils le rationnalisent en rappelant d'une part l'interdit, et d'autre part l'autorisation de l'instance médicale.

Les fantasmes d'adultère accompagnent le donneur dans son acte. Certains imaginent une ou des femmes, ou pensent précisément à des gens stériles de leur connaissance. Ils souhaiteraient alors obtenir le résultat des inséminations. Ce fantasme exprime le désir de "posséder" la femme d'un ami et aussi dans une dimension homosexuelle de prendre la place de l'ami qui ne peut rendre sa femme enceinte.

Le principe de l'anonymat et du secret garanti par le CECOS, sous-tendu par le fantasme d'adultère, constitue une limite stricte qu'il est impossible de dépasser. L'institution médicale représente l'instance de transgression possible mais qui interdit tout désir de confirmation réelle du fantasme : la seule confirmation étant la substitution du sperme (infécond) d'un homme par celui (fécond) du donneur.

\section{DES DIFFICULTES A RECRUTER DES DONNEURS}

Le recrutement des donneurs est en baisse constante au niveau national (Figure 1) depuis 1986 (année intermédiaire dans 


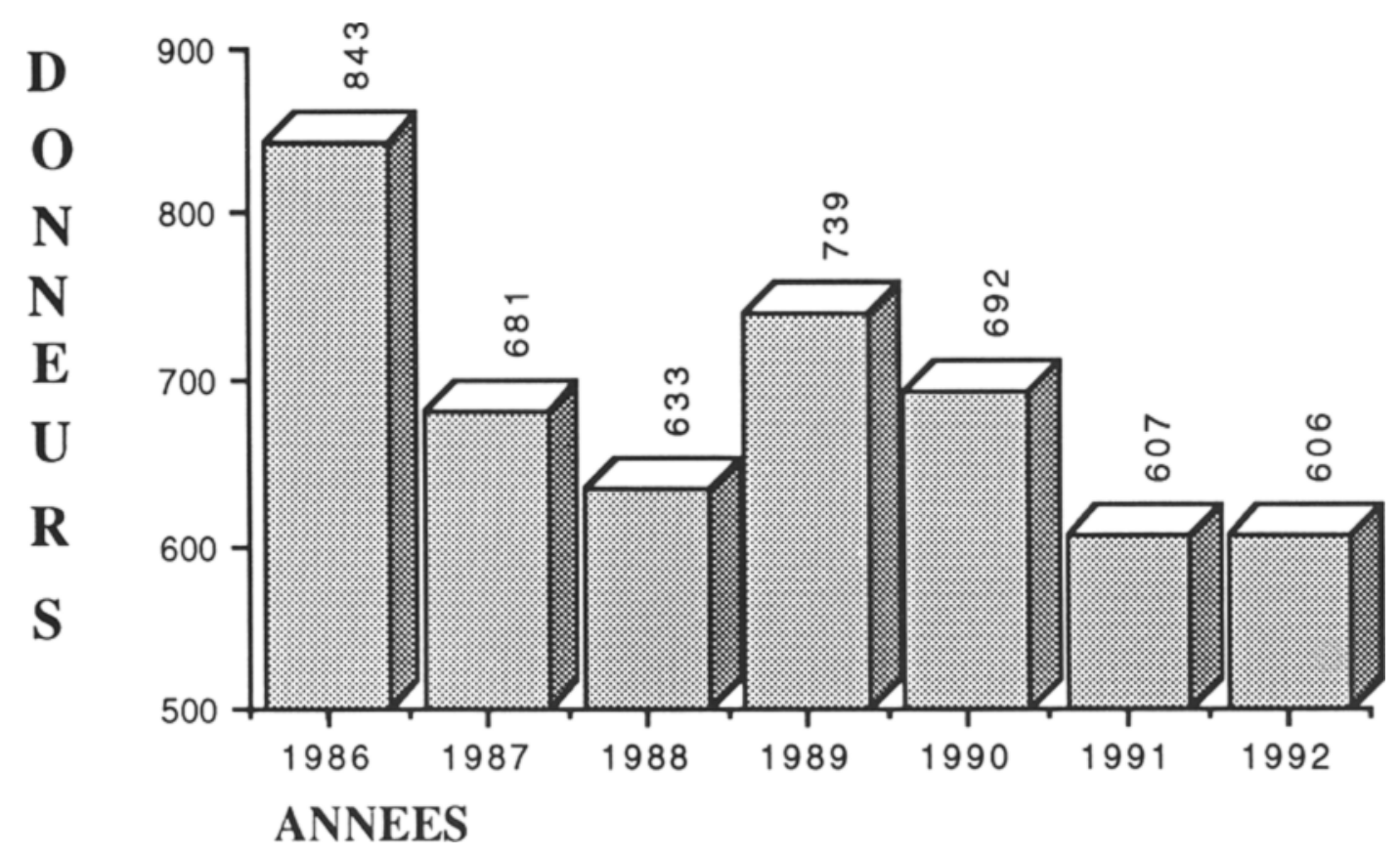

Figure 1 : Fédération des CECOS.

l'histoire des CECOS). Il est difficile d'émettre des hypothèses sur ce problème. Pour les années 1991-1992 (les chiffres de l'année 1993 ne sont pas connus à ce jour), l'affaire du sang contaminé a discrédité fortement l'institution médicale en général et par corollaire le don, dans la mesure où il est aussi organisé par une institution médicale. S'il est à remarquer que le nombre des demandeurs d'IAD ne diminue pas, au sens où ils ne semblent pas craindre une contamination, les hommes ne semblent pas enclins à faire don d'un produit d'euxmêmes au regard du manque de sérieux de la part de certains médecins. Il existe par ailleurs une confusion dans l'esprit du public entre le don d'organes et le don de produits qui se régénèrent. Les différents faits divers concernant des méthodes "abusives" de prélèvement d'organes et les prises de position de médecins sur les difficultés à se procurer des organes à transplanter sèment le trouble dans l'esprit du public. Il est fréquent de constater que des gens ne différencient pas le prélèvement d'organes (sur des cadavres) du don (de son vivant) de produits qui se régénèrent. L'institution médicale donne l'image de "vampire" qui peut mal utiliser les dons qu'elle suscite.

Les difficultés à recruter des donneurs sont liées au fait qu'un même sperme sert à obtenir cinq grossesses au maximum pour limiter les risques de rencontre entre les enfants du donneur et ceux conçus avec son sperme. Les CECOS sont dans l'obligation de recruter toujours de nouveaux donneurs. Ils ne peuvent donc pas constituer un fichier de personnes à solliciter périodiquement comme le pratiquent par exemple les centres de transfusion sanguine.

Les CECOS n'ont jusqu'à présent pas entrepris de mener une politique de recrutement des donneurs avec le concours de professionnels de la communication et de la publicité. Il y a seulement un an que la fédération des CECOS travaille pour établir un plan de communication avec l'élaboration de messages, la définition d'un public à sensibiliser. Ces opérations sont coûteuses et il n'y pas encore de consensus pour les financer. 
La principale méthode de recrutement employée par les CECOS est la demande formulée envers les couples en attente d'IAD d'adresser un donneur. Il est précisé que le don de cette personne servira à d'autres qu'à eux-mêmes. Cette méthode porte ses fruits dans la mesure où pour la majorité des CECOS, elle constitue quasiment l'unique moyen de recrutement, avec les résultats fluctuants que nous avons décrits.

Si un couple amène un donneur au CECOS, il voit son délai d'attente raccourci de plusieurs mois. Cette méthode favorise certains couples aux dépens d'autres qui ont choisi de ne pas divulguer leur projet. Le système de "récompense" peut inciter des couples à trouver par tous les moyens un donneur, ce qui risque de les déstabiliser dans leur démarche, s'ils avaient choisi le secret. Dans une histoire aussi chargée affectivement que la demande d'enfant, le CECOS ne devrait pas être en droit de faire peser une telle requête. Même si les praticiens des CECOS sont en majorité favorables à la divulgation de la conception par IAD, ce ne doit pas être de leur fait d'amener les couples dans une situation où la liberté de choix est réduite.

Une idée qui a cours dans les CECOS est de demander aux médecins qui pratiquent les IAD de sensibiliser leur clientèle au don de sperme et de recruter ainsi des donneurs. Cette méthode s'avère très infructueuse car ces médecins n'ont aucune motivation sauf peut-être d'espérer que la demande d'IAD de leurs clients aboutisse rapidement. Outre que les gynécologues ont des clients stériles, nous pensons qu'il n'entre pas dans leurs attributions de recruter des donneurs. De plus, lorsqu'une personne se rend chez un médecin, elle est soucieuse, même sans être très malade, de son état de santé personnelle et n'est pas réceptive au service demandé dans ce lieu.

L'idée de faire de la publicité pour le don de sperme dans les services hospitaliers de maternité se révèle un échec. En effet, au moment de la naissance de leur enfant, les couples sont focalisés sur leur situation personnelle qui est de faire sien ce nouveau-né. Notre étude sur les donneurs confirme que les pères ne sont pas réceptifs à la sollicitation des CECOS durant la période de la petite enfance de leurs enfants.

La sollicitation des personnels hospitaliers, toutes catégories confondues, se révèle aussi être un échec. Il est erroné de penser que les personnels qui travaillent dans le secteur de la santé sont plus réceptifs à la demande d'un service tel que le don de sperme que dans d'autres secteurs d'activité. D'ailleurs, le directeur d'un centre de transfusion sanguine nous informait que les collectes de sang sont très infructueuses dans les centres hospitaliers. Nous pensons que les personnels de santé "paient" suffisamment de leur personne en travaillant dans ce lieu pour ne pas en plus vouloir rendre un service à l'institution qui les emploie.

Les demandeurs de vasectomie constituent une part de la population des donneurs. Si les uns acceptent d'être donneurs avant l'intervention chirurgicale, les autres estiment contradictoire de donner leur sperme au moment où ils demandent une stérilisation. Tout se joue sur l'acceptation du médecin à pratiquer une intervention à la limite de la légalité sans imposer auparavant le don de sperme au motif que ces hommes ont la chance d'être fertiles et qu'ils doivent ainsi secourir des hommes stériles.

La liste des modes de recrutement n'est pas exclusive. Nous estimons primordial que le don de sperme soit librement consenti, en dehors de toute contrainte ou pression. C'est pourquoi, nous pensons que la priorité est l'élaboration d'un message incitatif sur le thème du don de sperme. Il est important aussi de ne pas attribuer au médecin un rôle de recruteur. La sollicitation doit sortir de la relation du médecin à sa clientèle. Il est donc opportun de s'ouvrir à un large public.

Depuis une dizaine d'années le CECOSLyon organise des opérations publicitaires 
au moyen d'articles rédactionnels dans la presse locale, distribution de tracts et envoi de lettres de sollicitation à des particuliers.

Le nombre de donneurs du CECOS-Lyon (Figure 2) subit la même baisse qu'au niveau national. Les fluctuations sont dépendantes de la réussite des opérations publicitaires. L'année 1986 a été particulièrement fructueuse. Le CECOS a adressé cette année-là une lettre de sollicitation aux abonnés du Nouvel Observateur des départements du Rhône et de l'Isère. Une opération similaire a été menée en 1989 et 1990 auprès d'adhérents de mutuelles de santé. Suivant le groupe socio-professionnel de référence, les donneurs qui se présentent au CECOS correspondent à $0,2 \%$ en moyenne des personnes sollicitées. L'année 1993 voit une hausse du nombre de donneurs, liée à une opération publicitaire (vingt donneurs pour le quatrième trimestre de l'année) qui se poursuit sur l'année 1994.

Il n'est pas aisé de sortir de la confidentialité pour aller vers une publicité du don de sperme. Nous remarquons depuis quelques années que des personnes sollicitées expriment d'emblée leur opposition à la procréation par IAD. Cette opinion se retrouve dans le sondage de l'institut Louis Harris [5], qui démontre que $46 \%$ contre $48 \%$ des personnes interrogées sont défavorables à la "procréation artificielle avec un donneur extérieur au couple". Ce sondage prouve que le public est informé et qu'il n'est pas indifférent à ce sujet (seulement $6 \%$ de personnes "sans opinion"). C'est pourquoi les résistances sont grandes à accepter de publier une information sur ce sujet.

Deux exemples récents : les Transports en Commun Lyonnais ont créé un espace publicitaire par projection de diapositives sur les quais du métro. Les publicités présentées sont commerciales, mais émanent surtout d'organismes humanitaires (Banque alimentaire, S.O.S. Amitié), de santé publique (informations sur le sida, la drogue). La demande du CECOS d'un espace publicitaire a été rejetée au motif que "la nécessaire neutralité d'un service publique n'est pas compatible avec le thème du "don de sperme" pouvant être sujet à controver-

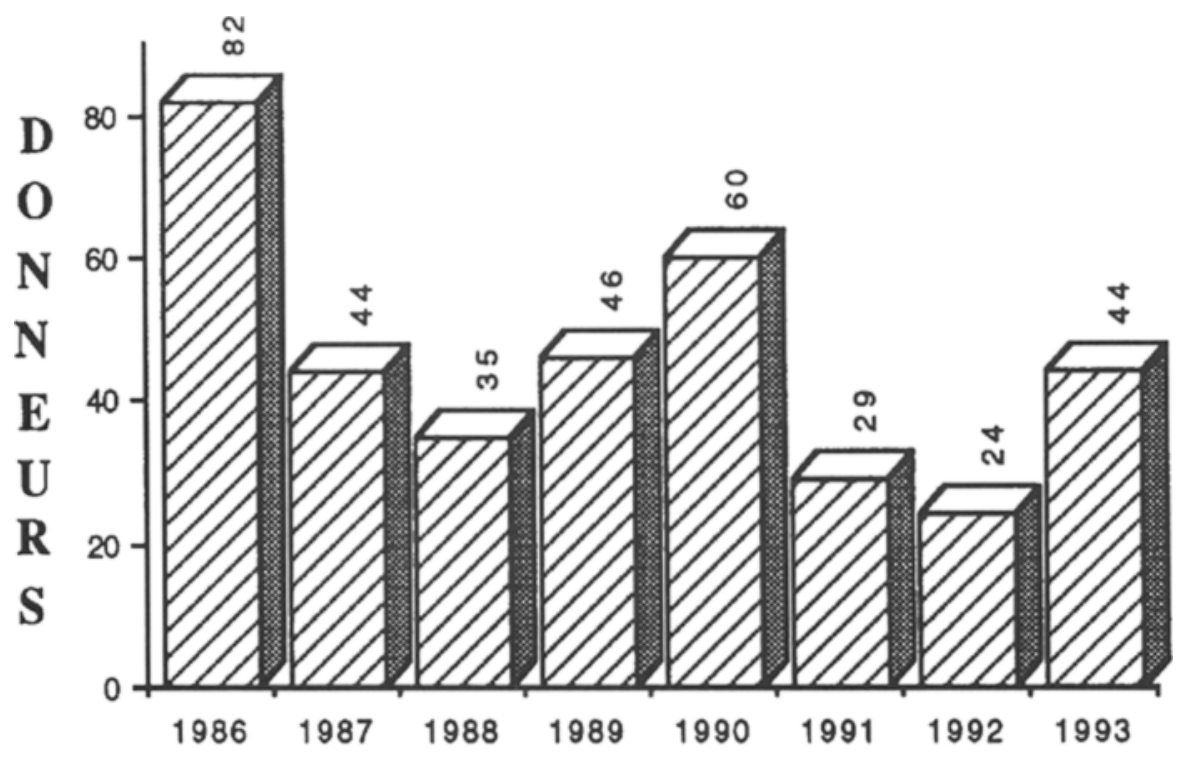

ANNEES

Figure 2 : Donneurs CECOS-Lyon. 
se". La demande du CECOS n'est pas moins neutre que l'information sur le sida ou la drogue, mais elle concerne directement la sexualité et la procréation. C'est sans doute la principale raison du refus avec peut-être aussi le désaccord sur le mode de conception par IAD des enfants. Le même refus nous a été opposé auprès de trois fédérations de parents d'élèves auxquelles nous demandions de faire parvenir le message du CECOS à leurs adhérents. Toutes trois ont refusé pour ne pas choquer leurs adhérents.

Les opérations publicitaires permettent de recruter (Figure 3 ) en moyenne $61 \%$ des donneurs (de $50 \%$ à $75 \%$ suivant les années). Les demandeurs de vasectomie constituent $29 \%$ des donneurs. Ils sont de moins en moins nombreux, ce qui correspond aussi pour les deux dernières années à une baisse importante des demandes de vasectomie de façon générale. Les donneurs adressés par des couples ou un membre de l'équipe du CECOS constituent $10 \% \mathrm{du}$ nombre total des donneurs.
Une image publicitaire (Dessin $n^{\circ} 1$ ) a été proposée par la fédération des CECOS en 1989. La formule "Vous avez le don de faire vivre" associée à l'image d'un poussin frâ̂chement éclos nous semblait percutante. Jouer sur la notion de don, c'est-à-dire avoir une capacité et pouvoir en faire profiter d'autres pour transmettre la vie, nous semblait atteindre son but. Mais la représentation graphique de la fleur sortant de l'œuf introduisait sans doute trop d'étrangeté dans la compréhension globale du message, à l'instar du donneur "étranger" au couple, qui est une formulation assez péjorative de l'IAD. L'utilisation de la métaphore en matière de communication est souvent incomprise car elle differre le contenu manifeste du message et oblige le lecteur à le décoder. Nous avons diffusé cette image et avons constaté qu'elle n'avait pas d'impact sur le public. C'est pourquoi, nous essayons maintenant de construire un message au plus près de la réalité de notre propos à transmettre pour que le public le comprenne immédiatement, sans effort de décryptage.

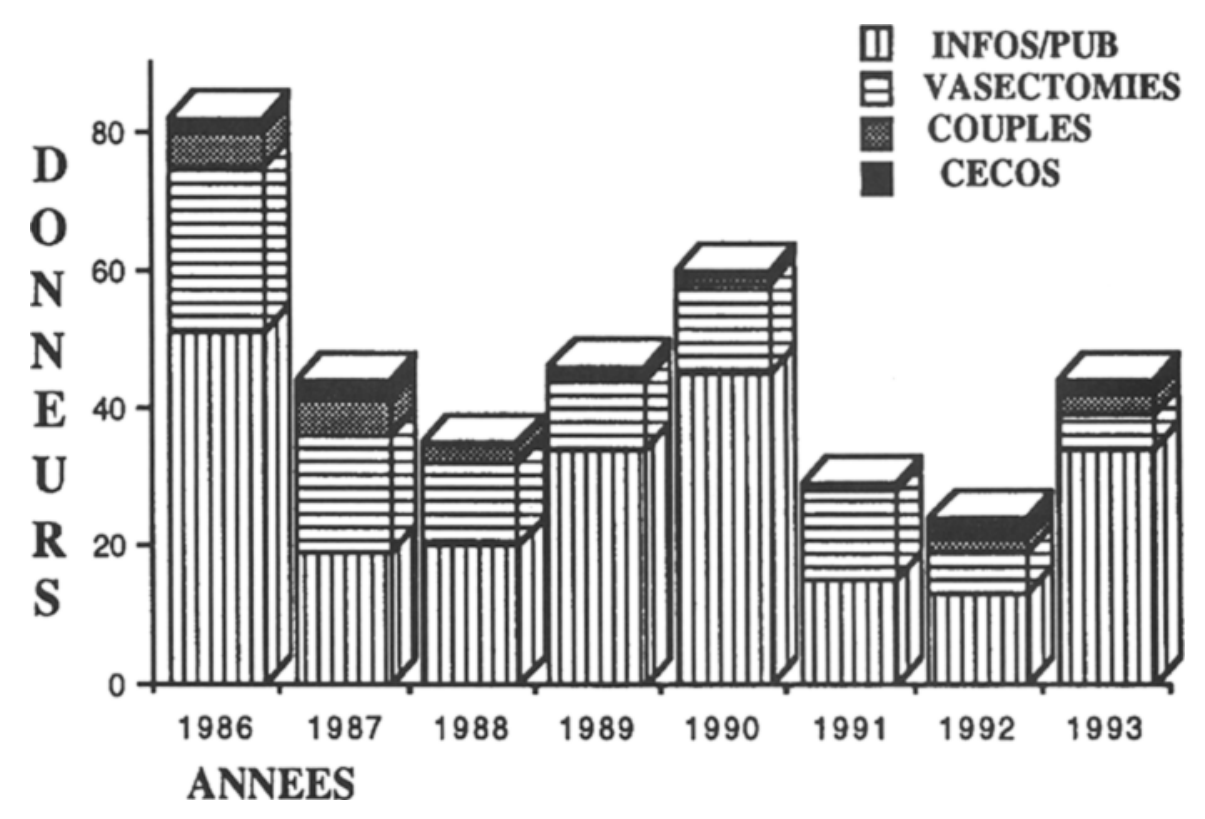

Figure 3 : Modes de sollicitation des donneurs CECOS-Lyon. 


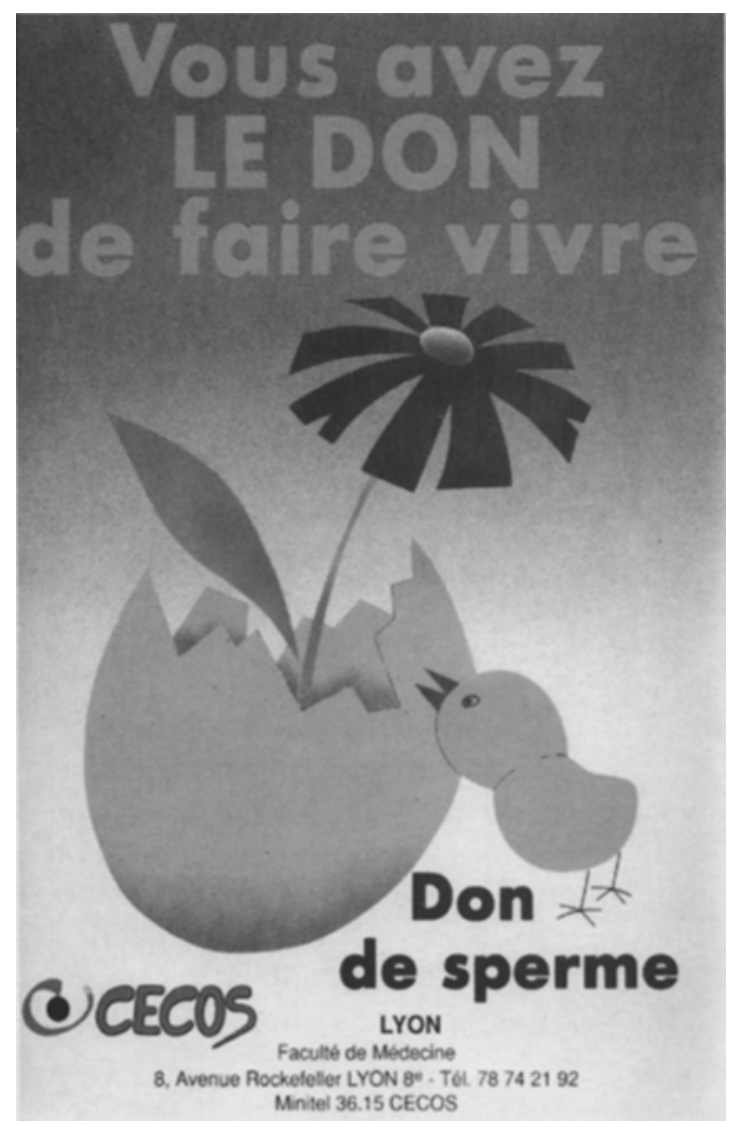

$\operatorname{Dessin} n^{\circ} 1$ :

Lors de sa dernière campagne d'information sur le don de sperme (quatrième trimestre 1993), le CECOS-Lyon a fait appel aux services du dessinateur Dubouillon dont les dessins humoristiques dans les quotidiens lyonnais et un hebdomadaire d'annonces de spectacles sont reconnus et appréciés du public. Nous avons écrit deux scénarios et confié la réalisation graphique à ce dessinateur. Deux bandes dessinées ont été réalisées, imprimées sous forme de tracts publicitaires et distribuées à un large public dans la rue. L'accueil du public a été chaleureux et attentif. Cette opération publicitaire confirme qu'il est possible de sortir de la confidentialité et de présenter le sujet sur un mode plaisant tel que Le Quotidien du Médecin le note dans un article intitulé : A Lyon : l'humour au service du don de sperme [7].

Le premier scénario (Dessin $n^{\circ} 2$ ) énonce différentes façons d'avoir un enfant et se termi- ne par l'IAD en informant du manque de donneurs de sperme. La première image fait référence à l'Annonciation avec une connotation iconoclaste à l'égard de Marie, première femme de l'Histoire devenue enceinte sans l'aide d'un homme. Les images suivantes alternent différentes compositions de famille. Ce scénario a pour fonction de mettre l'accent sur l'IAD comme étant le choix de certains couples stériles aux dépens d'une autre solution (l'adoption par exemple). L'image de la famille nombreuse est une incitation à l'attention des pères de considérer leur fertilité comme une possibilité d'être donneur.

Le deuxième scénario (Dessin $n^{\circ} 3$ ) est basé sur la conversation d'un couple dans un restaurant. Un homme annonce à sa femme qu'il a décidé de donner son sperme, suite aux confidences d'une amie sur la stérilité de son partenaire. Cette histoire se veut conviviale, proche du lecteur. Elle propose une identification avec le personnage principal présenté comme donneur potentiel. La notion de plaisir est énoncée avec un double sens : plaisir de donner anonymement du sperme, c'est-àdire imaginairement à une ou des femmes pour qu'elle soient enceintes et aussi en filigrane le plaisir de la transgression. Le personnage du serveur du restaurant cristalise les résistances au don de sperme mais il se laisse peut-être convaincre et entrevoit l'éventualité d'être donneur lui-même.

Les deux scénarios sont intitulés Histoire d'enfant pour signifier que le don contribue à la conception d'enfants. Il semble important en effet d'insister sur les données du problème, quitte à démobiliser un nombre important d'hommes au profit de ceux qui souhaitent que des enfants soient conçus avec leur sperme et en dehors de leur couple. La référence du CECOS est précédée de la mention Don de spermatozoïdes afin d'atténuer la dimension érotique et sexuelle du sperme. Néanmoins, le mot sperme apparaît dans le courant de l'histoire pour tenter de lever toute ambiguïté sur le service qui est demandé. Mais cette ambiguïté demeure pour certains hommes qui imaginent que leurs sper- 


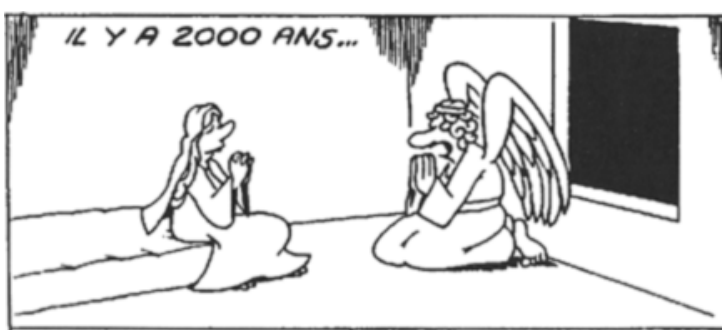

IL Y A CEUX POUR DUICE N'EST PAS UN PROBLEME..

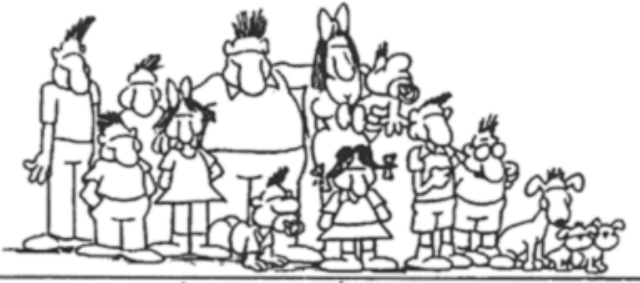

IL Y A CEUX QUI VONT AU PE'ROW ADCOER UN ENFANT",
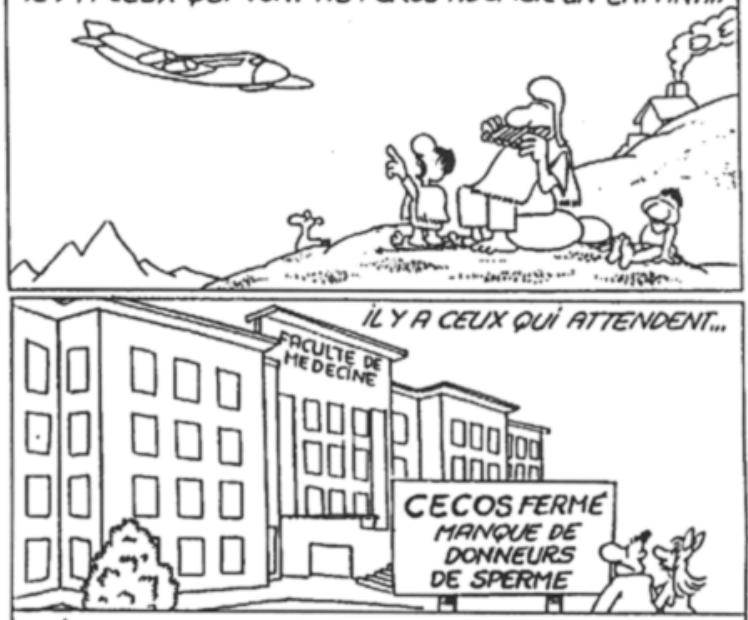

ILS ATIENDENT VOTRE DON POCR AVOIR UN ENFANT.
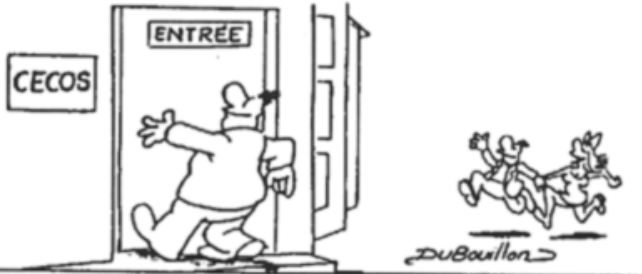

\section{DON DE SPERMATOZOÏDES}

CFCOS-FACULTE dE MEDECINE

Centre d'Etude et de Conservation du Sperme humain

898 $78777156 \quad(24$ H/ 24 H)
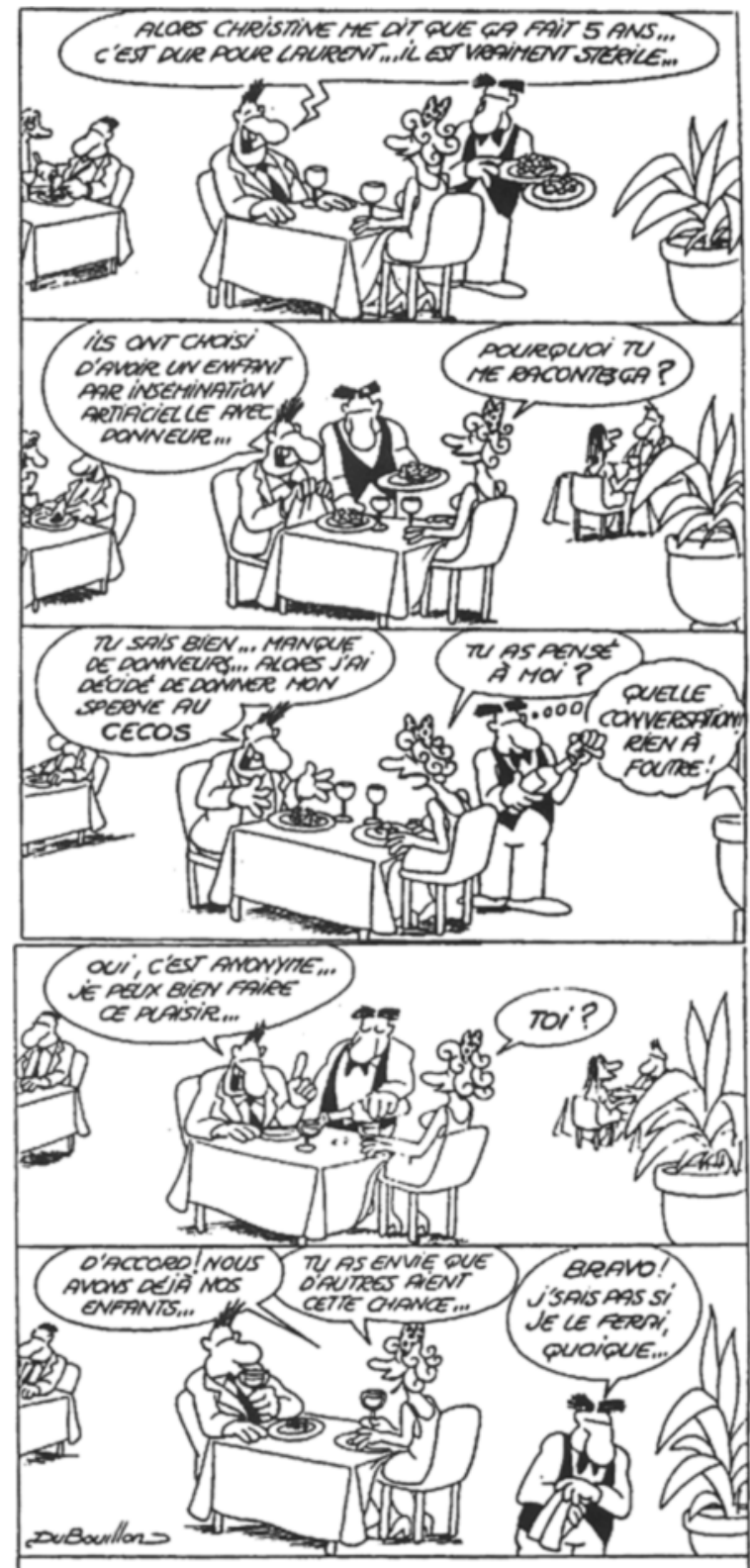

\section{DON DE SPERMATOZOIIDES}

CECOS-FACULTE dE MEDECINE

Centre d'Etude et de Conservation du Sperme humain

898 $78777156(24 \mathrm{H} / 24 \mathrm{H})$

\section{Dessin $n^{\circ} 2$ :}

$\operatorname{Dessin} n^{\circ} 3$ : 
matozoïdes, voire peut-être leur sperme, peuvent être directement prélevés dans le scrotum par le médecin.

\section{CONCLUSION}

Il est et sera toujours difficile de recruter un nombre suffisant de donneurs de sperme pour permettre un fonctionnement satisfaisant de la pratique de l'IAD. La contestation de la procréation avec un tiers donneur est importante telle que le révèlent les différents sondages d'opinion depuis une dizaine d'années. Nous constatons aussi les difficultés à définir un cadre légal à cette pratique médicale. Plusieurs projets et propositions de loi ont été élaborés depuis plusieurs années sans qu'à ce jour, un consensus se soit dégagé parmi les instances gouvernementales et parlementaires, tant le sujet divise l'opinion.

La procréation par IAD remet en cause l'établissement de la paternité. Même si celle-ci est toujours présumée, elle n'en est pas moins définie comme étant biologique et sociale. $\mathrm{La}$ procréation par IAD énonce clairement que cette paternité-là n'est que sociale. Par corrolaire, les donneurs de sperme peuvent légitimement penser qu'ils ont des enfants qui sont nés dans une autre famille que la leur et pour lesquels aucun lien ne leur est reconnu.

Les fantasmes d'inceste entre enfants constituent une limite au don qu'il est impossible de contourner. Les donneurs acceptent cette hypothèse mais nous ignorons souvent l'impact de leur geste dans leur vie personnelle et surtout plusieurs années après leur don. Ainsi, nous avons rencontré récemment un ancien donneur venu au CECOS lorsque ses enfants avaient dix et huit ans. Demandeur d'une vasectomie dix ans après son don, il remettait en cause et regrettait son geste au moment où ses enfants devenaient adultes. Il était véritablement anxieux devant la portée de son acte qui engageait l'avenir de ses enfants et il craignait leur rencontre avec ceux conçus avec son sperme. Il disait n'avoir pas pensé à cette éventualité lorsqu'il était venu donner son sperme pour rendre service à des couples stériles.

\section{REFERENCES}

1. AUROUX M. : "Age du père et développement", Contraception-Fertilité-Sexualité, 1993, Vol. 21, $n^{\circ} 5$ : pp. 382-385. Cf. aussi : THEPOT F., WACK T., SELVA J. et al., "Age paternel et issues de grossesses : expérience des CECOS", ContraceptionFertilité-Sexualité, 1993, Vol. 21, n5 : pp. 388-390.

2. CLEMENT J.L. : "Profil psycho-sociologique d'une série de 850 donneurs de sperme", ContraceptionFerwtilité-Sexualité, 1993, Vol. 21, nº 6 : pp. 498-500.

3. CLEMENT J.L., DELORME F. : "Le don de sper-me?", L'évolution psychiatrique, 57, 3 : 1992, pp. 405-415.

4. DAVID G. : "L'insémination artificielle et le système CECOS", CECOS, L'insémination artificielle, Paris, Masson, 1991, $229 \mathrm{p}$.

5. L'Express, 26 janvier-1er février 1990.

6. PERNOT J.F., SAINT-POL P. : "Etude préliminaire des résistances psychologiques au don de sperme", Contraception-Fertilité-Sexualité, 1989, Vol. 17 : n 7-8, pp. 669-671.

7.Le Quotidien du Médecin, 4 octobre 1993.

\section{ABSTRACT \\ Sperm Donation}

Jean-Loup CLEMENT

\section{CECOS, Faculté de Médecine, Lyon}

Since its creation, the recruitment of donors has been a constant difficulty of the CECOS. Donors have to be sollicited directly or indirectly. The limited number of pregnancies from the same donor obliges the CECOS to recruit constantly new ones. They cannot call for the same donors periodically.

Sperm donation, like other human products, should be done with the free consent of the donors. Its aim is to allow the conception of children outside the family organization of the donor. This motivates some men to accept donation and at the same time démotivates many of them. Hence the necessity of advertisment for sperm donation to favor recruitment among a large population.

Key-words : sperm donation, recruitment of donors, advertising. 